International Journal of Current Microbiology and Applied Sciences

ISSN: 2319-7706 Volume 6 Number 2 (2017) pp. 817-822

Journal homepage: http://www.ijcmas.com

Original Research Article

http://dx.doi.org/10.20546/ijcmas.2017.602.091

\title{
Sporotrichosis Centering Siliguri and its Sub-Himalayan Neighbours
}

\author{
Haldar Nibedita ${ }^{1^{*}}$, Haldar Niladri ${ }^{2}$, Chakrabarti Indranil ${ }^{3}$ and M.K. Sharma ${ }^{4}$ \\ ${ }^{1}$ Department of Microbiology, MGM Medical College and LSK Hospital, Kishanganj, Bihar and \\ "The Microbes" Pathological Laboratory, Siliguri, Darjeeling, India \\ ${ }^{2}$ Department of Pathology, D Y Patil Medical College, Pimpri, Pune \\ BPKIHS, Dharan, Nepal \\ ${ }^{3}$ Department of Pathology, North Bengal Medical College, Siliguri, Darjeeling, \\ West Bengal, India \\ ${ }^{4}$ Sharma's Skin Foundation, Siliguri, Darjeeling, West Bengal, India \\ *Corresponding author
}

A B S T R A C T

\begin{tabular}{|l|}
\hline K e y w o r d s \\
Sabouraud dextrose \\
agar (SDA), \\
Lactophenol \\
cotton blue (LCB), \\
Sporotrichosis, \\
Siliguri.
\end{tabular}

\section{Keywords}

Sabouraud dextrose agar (SDA),

Lactophenol

Sporotrichosis,

Siliguri.
Sporotrichosis is a sub-acute or chronic granulomatous fungal infection involving primarily the skin and subcutaneous tissue with neighbouring lymphatics caused by a dimorphic fungus Sporothrix schenckii. The disease is worldwide in distribution. In India sporotrichosis is endemic in the Sub-Himalayan region; stretching from West Bengal, Assam and Manipur in the North East and Himachal Pradesh in the North West. In comparison to extensive studies at far eastern states of Assam and Manipur, till now very few studies are there in Siliguri (a small Sub division at the foothills of Himalaya within District Darjeeling of West Bengal) and surrounding areas. Therefore a retrospective study has been carried out over a period of nine years since $1^{\text {st }}$ Dec, 2007 up to $30^{\text {th }}$ Nov, 2016 on 150 patients with skin lesions attending Sharma's Skin Foundation at Siliguri. All the samples from skin lesions were tested at "The Microbes pathology lab", Siliguri. Out of 40 culture positive cases interestingly $16(40 \%)$ of patients were permanent inhabitants of Siliguri and neighbouring plains like Naxalbari, Phansidewa, Jalpaiguri, Haldibari etc., not migrants from the surrounding hilly areas. Twenty four $(60 \%)$ of the patients are belonged to hilly area e.g., Sikkim, Darjeeling, Kalimpong, Kurseong and Mirik. Lymphocutaneous variants were the predominant forms e.g. $26(65 \%)$ followed by fixed cutaneous variants $13(34 \%)$ and in $1 \%$ there were disseminated cutaneouos lesions scattered all over the chest, arms and thighs. One case had been presented as Pyoderma gangrenosa, a moribant case cured by giving IV amphotericin B. The chances of isolating Sporothrix schenckii from pus samples and wound swabs from the lesions were very good giving confirmed diagnosis in 10 to 14 days. Some of the positive samples $2(5 \%)$ were sent to referral centres at Delhi for serology by Latex agglutination tests giving positive results with significant antibody titres. All the patients were successfully treated with saturated solution of potassium iodide. The cases reported here indicate that the disease is prevalent in Siliguri with surrounding plains and hills. It may be another emerging endemic area in the Sub-Himalavan belt.

\section{Introduction}

Sporotrichosis is a chronic, pyogranulomatous fungal infection of cutaneous and sub cutaneous tissues, which remain localized or may show lymphatic spread with occasional 
dissemination to other parts of body. This is caused due to inoculation of skin by Sporothrix schenckii, a thermally dimorphic environmental saprotrophic fungus, which has a worldwide distribution. The disease is also called as "Rose Gardener's disease" (Text Book of Mycology by Jagdish Chander).

The disease was originally described in 1898 by a medical student, Benjamin Schenck, at Johns Hopkins Hospital in Baltimore, USA. In 1932, Ghosh reported the first case of sporotrichosis from Kolkata, which represented endemic focus in northeast belt of India.

Padhye and colleagues reported Indian case from Chandigarh in 1992. It is imperative to understand the epidemiology of sporotrichosis as an accurate diagnosis, and prompt initiation of the appropriate treatment will prevent chronic debility. This is the first extensive compilation of culture proven cases of sporotrichosis from Siliguri, District Darjeeling, India. It thus justifies our interest in analyzing data from our laboratory seen over 9 years briefly describing the clinical aspects and cultural characteristics of the isolates obtained from the clinical specimens.

\section{Materials and Methods}

A retrospective study has been carried out over a period of nine years since $30^{\text {th }}$ Dec, 2007 up to $30^{\text {th }}$ Nov, 2016 on a total number of 150 patients with various skin lesions attending a Dermatology clinic - ("Sharma's Skin Foundation") at Siliguri. All the samples of pus, exudate and skin swabs from the lesions were processed and tests were done at "The Microbes pathological laboratory", Siliguri. Total number of skin samples received with the clinical possibility of Sporotrichosis: 150. Suggestive history of inoculation: 105 (70\%) (History of trauma with thorns, straw, hay, wood splinters, needles, close association with pets like cattle, cats etc.). No suggestive history: 45 (30\%). Clinical types (Fig. 1-7): Lymphocutaneous variety: predominant forms 89 (60\%), Fixed cutaneous forms: 60 (40\%), Disseminated cutaneous lesion: 1 case (pyoderma gangrenosa). Pus samples and wound swabs from the lesions: Processed in the routine mycology lab (The Microbes pathology lab, Siliguri) for direct microscopy and culture.

\section{Mycological examination}

I. Gram stain of the fluid aspirates followed by II. Culture of the samples: cultured on two sets of Sabouraud's dextrose agar (SDA) with gentamicin $0.02 \mathrm{mg} / \mathrm{ml}$, chloramphenicol 0.05 $\mathrm{mg} / \mathrm{ml}$ and cycloheximide $0.5 \mathrm{mg} / \mathrm{ml}$; one of each set: incubated at $25^{\circ} \mathrm{C}$ and $37^{\circ} \mathrm{C}$. Colony characteristics observed and Lacto phenol cotton blue mount (LCB) of the growth done to see the morphological characteristics of the colony. III. Slide culture done to confirm the findings. IV. Conversion to yeast phase: demonstrated by subcultures on brain heart infusion blood agar with gentamicin 0.02 $\mathrm{mg} / \mathrm{ml}$, Chloramphenicol $0.05 \mathrm{mg} / \mathrm{ml}$ and cycloheximide $0.5 \mathrm{mg} / \mathrm{ml}$. Incubated at $37^{\circ} \mathrm{C}$.V. Sera of some of the positive patients (5\%). Sent to referral centres at Delhi for Latex agglutination test using the kit LASporo Antibody System (ImmunoMycologics, USA). VI. Cytological examination done with the aspirate from the lesion stained with Leishman stain.

\section{Results and Discussion}

Out of 40 culture positive cases 16 (40\%) were permanent inhabitants of Siliguri and surrounding plains e.g., Naxalbari, Phansidewa, Jalpaiguri, Islampur, Haldibari. Non-migrants from the surrounding hilly areas. Area wise distribution of these 16 cases: Siliguri 8, Jalpaiguri 3, Naxalbari 2, Phansidewa 1, Haldibari 1, Islampur 1. Out of 
40 culture positive cases $24(60 \%)$ belonged to hilly region of Sikkim, Darjeeling, Kurseong and Mirik. Areawise distribution of these 24 cases: Sikkim 14, Darjeeling 5, Mirik 3 and Kurseong 2.

I. Gram stain of the fluid aspirates: non contributory.

II. Colony characteristics of the growth on SDA at SDA: White to grayish colonies with aerial hyphae: observed after $7-10$ days of incubation on SDA at $25^{\circ} \mathrm{C}$, eventually turned greenish black on further incubation. LCBs mount of the growth: Thin, hyaline, septate hyphae with pyriform conidia arranged in a bouquet-like pattern around erect conidiophores and also directly arising from the hyphae.

The chances of isolating Sporothrix schenckii: very good giving confirmed diagnosis in $10-$ 14 days.

III. Slide culture: showed similar LCB findings as those found from growth at SDA kept at $25^{\circ} \mathrm{C}$.

IV. Conversion to yeast phase: Growth on SDA at $37^{\circ} \mathrm{C}$ showed Moist, smooth, creamish colonies observed after 3 - 6 days. Gram stain from the growth revealed Grampositive spherical budding yeast cells.

V. Serological test: Sera of some of the positive patients (5\%). Sent to referral centres at Delhi for Latex agglutination test using the kit LA- Sporo Antibody System (ImmunoMycologics, USA). All the samples showed positive result by latex agglutination test (Titre: 1: 8 and 1: 16).

Fig.1 Growth of Sporothrix schenckii on SDA at $25^{\circ} \mathrm{C}$

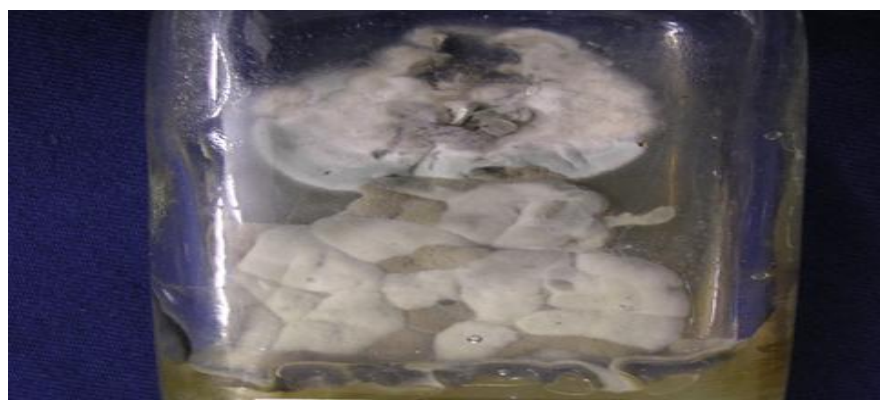

Fig.2 and $3 \mathrm{LCB}$ mount of fungal growth on SDA at $25^{\circ} \mathrm{C}$
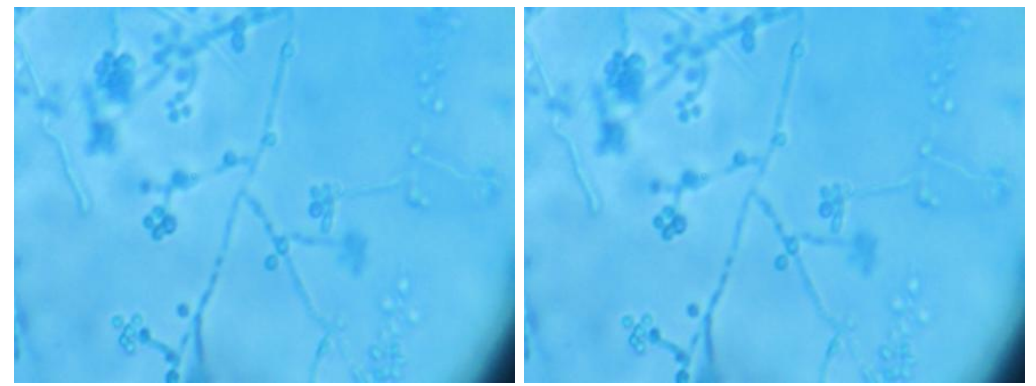
Fig.4 A. Slide culture B. Fungal growth on blood agar at $37^{\circ} \mathrm{C}$

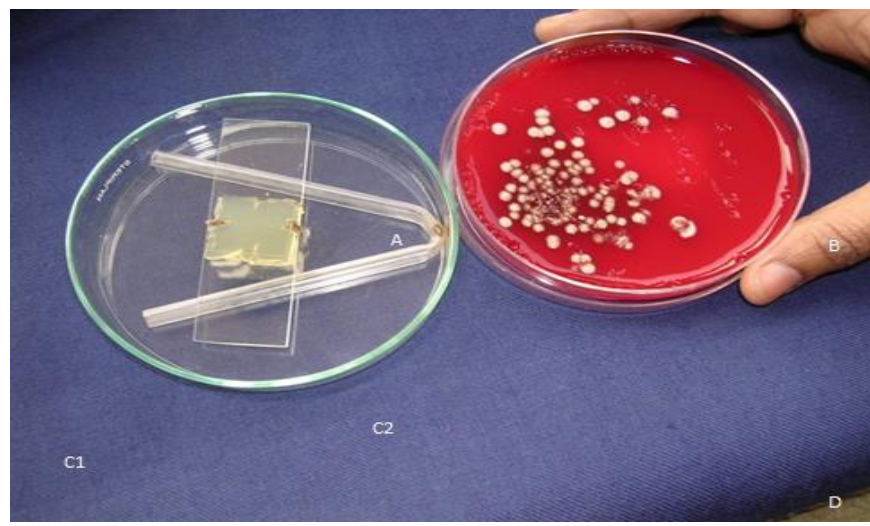

Fig.5 LCB mount of yeast phase of Sporothrix schenckii

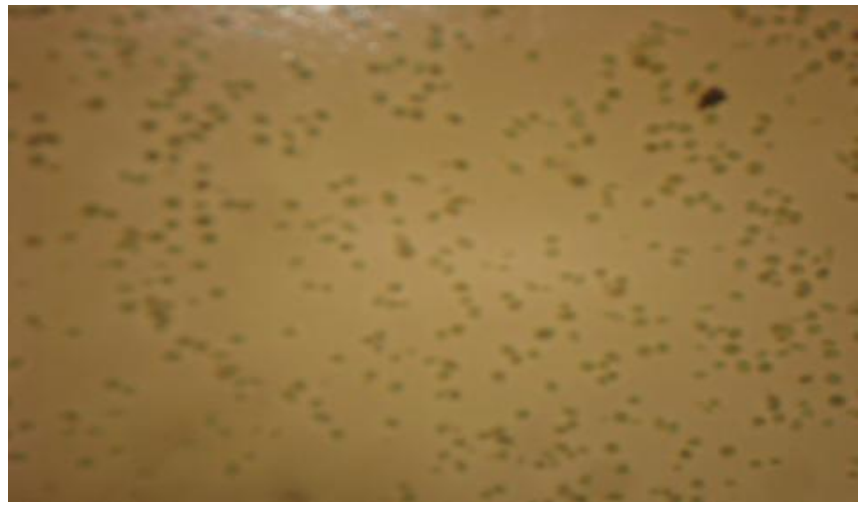

Fig.6 Lymphocutaneous sporotrichosis of forearm

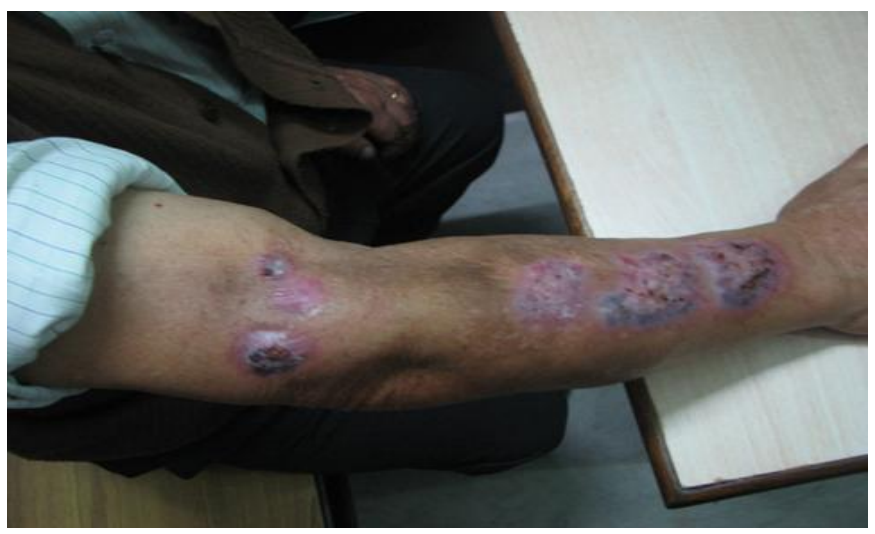


Fig.7 Lymphocutaneous sporotrichosis of leg (Above) Before treatment (Below) After treatment

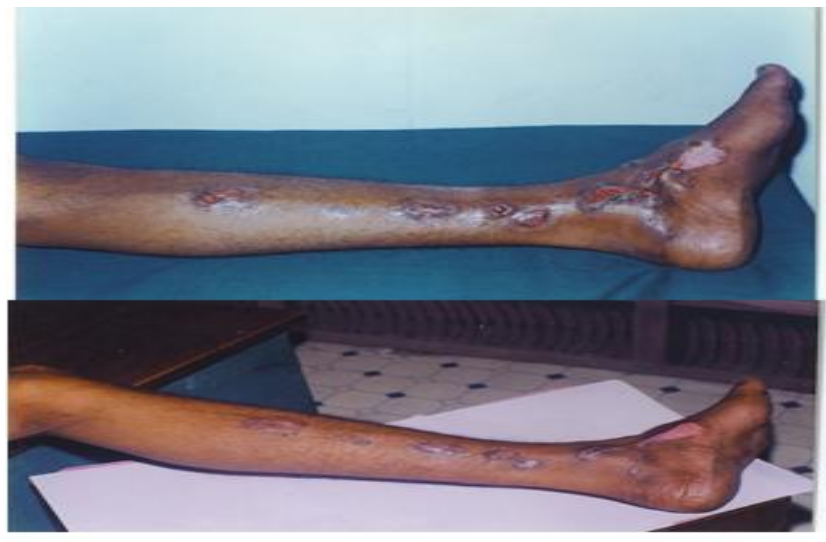

Figure 8 and 9 Fixed cutaneous variety of sporotrichosis

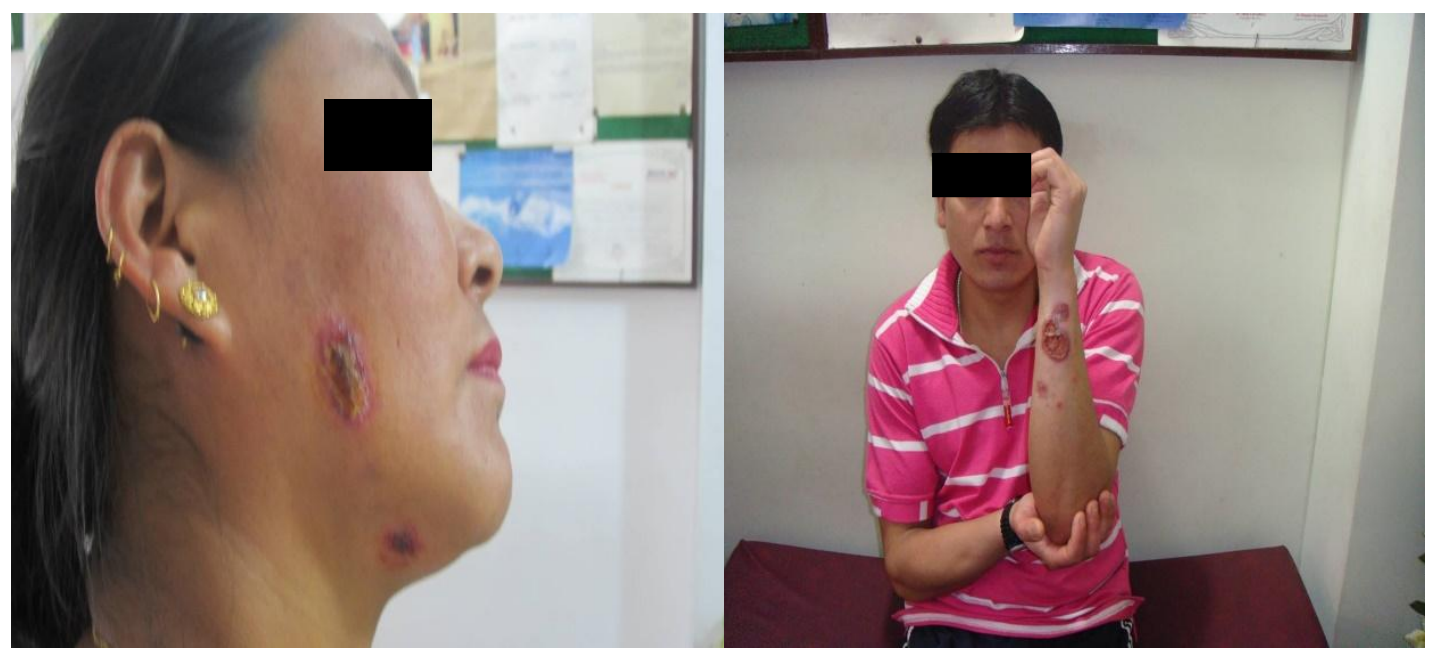

VI. Cytological examination done with the aspirate from the lesion stained with Leishman stain:

Showed mixed cell granulomas admixed with non-specific inflammatory cells. Yeast cells were not observed.

Garden soil samples of $10 \%$ of the culture positive patients tested did not yield any isolate of S. Schenckii.

Sporotricosis is prevalent more frequently in tropical and subtropical areas in climatic condition of high humidity (65\%) and moderate temperature of $25-28^{0} \mathrm{C}$. The exact environmental niche of $S$. Schenckii is not yet known but it is found as saprotroph associated with plants and soil. It is isolated from various natural sources and dead vegetation such as wood, bark, leaves, straw, sphagnum moss ad soil. The fungus is commonly found on decaying vegetation and is introduced into skin following minor trauma (Text Book of Mycology by Jagdish Chander).

Siliguri is the second largest city of West Bengal - situated in the plain at the foothills 
of "The Himalayas" at the latitude of $26.72^{0} \mathrm{~N}$ and the longitude of $88.43^{\circ} \mathrm{E}$. It has a hot and humid climate, but cool wind blowing from the Himalayas provides respite. The annual average rainfall ranges from $2600 \mathrm{~mm}$ to $4000 \mathrm{~mm}$. There is dense fog with light rain during winter. All these conditions of climate are suitable for the growth of the fungus Sporothix schenckii. This study reported the first case of this disease from the hilly eastern state of Sikkim and four additional cases including three from Darjeeling and one from Coochbehar District in the North-eastern part of West Bengal in the year 2007 (Haldar et al., 2007). $70 \%$ of our positive cases had a definite history of trauma with thorns, straw etc. $60 \%$ of our cases had lymphocutaneous variety which correlate well with study of Mahajan et al., (2014) who also found lymphocutaneous variety to be the commonst. microscopic detection of $S$. Schenckii in biopsy sections and Pus is always difficult (Sanyal et al., 1973; Kaufman, 1999) as also observed in our case. Though the fungus could not be recovered from the soil samples collected from the garden soils of $10 \%$ of the patients, the possibility of patients acquiring infection from soil or garden manure in their environment cannot be excluded. All the cases were successfully treated with saturated solution of potassium iodide. Kaufman and Chakrabarti et al., (1994) have proved that Saturated solution of potassium iodide is still the treatment of choice for sporotrichosis in developing countries. Many more cases of Spootrichosis possibly occur in these areas but are not detected due to lack of awareness and laboratory facilities. Therefore Siliguri and the surrounding Sub-Himalayan areas may be another emerging endemic area of sporotrichosis.

\section{References}

Chakrabarti, A., Roy, S.K., Dhar, S., Kuma, B. 1994. Sporotrichosis in north-west India. Indian J. Med. Res., 100: 62 - 65.

Haldar, N., Sharma, M.K., Gugnani, S.C. 2007. Sporotrichosis in North-East India. Mycoses, 50: 201 - 204.

Kaufman, C.A. 1999. Sporotrichosis. Clin. Infect. Dis., 29: 231-6

Mahajan, V.K. 2014. Sporotrichosis: an overview and Theapeutic options. Dermatol. Res. Practice, Article ID 272376, 13 pages.

Padhye, A.A., Kaufman, L., Durry, E., et al. 1992. Fatal pulmonary sporotrichosis caused by Sporothrix schenckii var. luriei in India. J. Clin. Microbiol., 30: 2492-4.

Sanyal, M., Basu, N., Thammaya, A., Tutakne, M.A., Gaind, M.L. 1973. Subcutaneous sporotrichosis in India. Indian J. Dematol. Venerol. Leprol., 39: 88-91.

Text Book of Mycology by Jagdish Chander.

\section{How to cite this article:}

Haldar Nibedita, Haldar Niladri, Chakrabarti Indranil and Sharma, M.K. 2017. Sporotrichosis Centering Siliguri and its Sub-Himalayan Neighbours. Int.J.Curr.Microbiol.App.Sci. 6(2): 817822. doi: http://dx.doi.org/10.20546/ijcmas.2017.602.091 